\title{
La influencia de la manipulación del tiempo en la complejidad, corrección y fluidez lingüísticas de tareas escritas en lengua extranjera
}

\author{
Irini Mavrou - Universidad Antonio de Nebrija, Madrid \\ emavrou@nebrija.es \\ Claudia Mariella Villar - Universität Mannheim, Alemania \\ cvillar@mail.uni-mannheim.de
}

Rebut / Received: 1-10-15

Acceptat / Accepted: 12-4-16

Resum. La influència de la manipulació del temps en la complexitat, correcció i fluïdesa de tasques d'escriptura en llengua estrangera. L'objecte d'aquest estudi va ser observar l'efecte de la manipulació del temps donat per a la realització de dues tasques d'escriptura en les àrees lingüístiques de complexitat, correcció i fluïdesa d'un grup de 65 aprenents grecs de llengua espanyola, així com les probables diferències entre el discurs natiu i el no natiu. Els resultats obtinguts van permetre concloure que donar temps als aprenents de segona llengua en els processos d'escriptura fomenta la complexitat sintàctica i la fluïdesa, encara que no sembla ser decisiu per a la correcció lingüística. Tanmateix es va observar que els parlants natius van elaborar textos amb més fluïdesa i complexitat sintàctica possible conseqüència del seu gran ventall de vocabulari i pel seu major recolzament en el coneixement implícit de la seva llengua materna que potencia un accés i una recuperació de la informació més ràpids i eficients.

Paraules clau: expressió escrita, complexitat cognitiva de la tasca, complexitat sintàctica, correcció, fluïdesa.

\begin{abstract}
The impact of manipulating time-on-task on linguistic complexity, accuracy and fluency in foreign language written production. The purpose of this study was twofold: first, to examine the impact of manipulating the time allowed for the completion of two written tasks on the linguistic complexity, accuracy and fluency of 65 Greek learners of Spanish as a foreign language; and, second, to examine potential differences between native and non-native speakers'
\end{abstract}


performances in terms of syntactic complexity and fluency. The findings led to the conclusion that providing students with sufficient time to engage in text production can foster syntactic complexity and fluency, but may not be conducive to greater linguistic accuracy. Moreover, it was found that native speakers seem to produce more fluent and syntactically elaborated texts. This result could be partly attributed to the wide range of vocabulary that native speakers have at their disposal, as well as to the implicit knowledge of their mother tongue, which ensure faster and more accurate knowledge access and retrieval processes.

Keywords: written production, cognitive task complexity, syntactic complexity, accuracy, fluency.

\section{Introducción}

En el ámbito de Adquisición de Segundas Lenguas (ASL) un amplio número de estudios se ha ocupado del impacto de la manipulación de las características de las tareas en la actuación del aprendiente de lengua extranjera (LE). Los principales representantes de esta línea de investigación han sido Robinson (2001, 2003, 2007), y Skehan (1996, 1998). Ambos autores reconocen que la complejidad cognitiva de las tareas se puede manipular externamente. Para Skehan (1996, 1998), el objetivo de tal manipulación es propiciar las condiciones necesarias para la distribución equilibrada de los recursos atencionales hacia el significado y la forma. Robinson (2001, 2003, 2007), por su parte, asume que la LE se adquiere a través del esfuerzo del aprendiente por atender a los requerimientos conceptuales que imponen las tareas mientras va aumentando su complejidad cognitiva.

Todo ello se refleja en los procedimientos de intervención pedagógica que sugieren los autores. Según Skehan (1996, 1998), la secuenciación de las tareas se debe hacer en función de las exigencias comunicativas y el esfuerzo cognitivo que suponen, mientras que Robinson $(2001,2003,2007)$ recomienda la secuenciación a lo largo de dos dimensiones denominadas resource-directing y resource-depleting ${ }^{1}$, lo cual, según el autor, posibilita el aprendizaje acumulativo y fomenta los procesos de automatización.

A pesar de las diferencias señaladas, los modelos teóricos propuestos por estos autores presentan ciertas similitudes con respecto a las predicciones que hacen sobre los efectos de la manipulación cognitiva de las características de las tareas en la actuación lingüística. Robinson sugiere que el esfuerzo por producir un lenguaje más complejo (resultado

\footnotetext{
1. Según el Triadic Componential Framework (Robinson, 2001, 2003), el aumento de la complejidad cognitiva en la dimensión resource-directing canaliza los recursos atencionales hacia los aspectos del código y promueve el desarrollo de conexiones entre función y forma. En cambio, la manipulación de las variables de la dimensión resource-depleting incrementa las demandas en los mecanismos de programación y de task switching. Por tanto, mediante la práctica consistente de tareas complejas, el aprendiente irá adquiriendo un mayor control y un acceso más rápido al conocimiento que ya dispone de la LE.
} 
del aumento de la complejidad cognitiva a lo largo de la dimensión resource-directing) conduce a un deterioro de la fluidez, predicción que concuerda con los postulados de Skehan, quien sostiene que habrá trade-offs (relación inversa, competición) entre el significado y la forma, entre fluidez y complejidad y/o corrección lingüísticas.

Robinson también coincide con Skehan en que el aumento de la complejidad de la tarea impone mayor carga cognitiva y compromete los recursos atencionales, aunque, según el primero, esto es cierto cuando se manipulan las variables de la dimensión resource-depleting. Esto significa que factores como la presión de tiempo, la realización de tareas duales o de tareas que no poseen una estructura clara conducen a una dispersión de los recursos cognitivos, afectando negativamente la complejidad, corrección y fluidez lingüísticas, y esto es precisamente lo que defiende Skehan, que si las exigencias cognitivas de las tareas son altas, las tres dimensiones de la lengua se verán perjudicadas ${ }^{2}$.

En los estudios que se adscriben a la línea de manipulación cognitiva de tareas —especialmente en aquellos que se han ocupado de la expresión escrita-, una variable ampliamente investigada ha sido la planificación del discurso. A pesar de la gran utilidad de la planificación en la expresión escrita, es importante señalar que esta no constituye un requisito sine qua non para la producción de un texto de calidad. Por ejemplo, en varios estudios, la planificación - $\mathrm{y}$ en concreto la planificación previa - se ha examinado a partir de tareas narrativas basadas en material gráfico. La utilidad de la planificación en este caso podría ser discutible, puesto que dichas tareas son poco demandantes a nivel de conceptualización del mensaje, es decir, liberan de la necesidad de generar, estructurar y organizar contenido nuevo. Además, el tiempo concedido para la planificación podría ser percibido como una larga espera, especialmente por parte de aquellos aprendientes que poseen una amplia experiencia en el aprendizaje de idiomas y cuentan con esquemas desarrollados sobre la estructura del género narrativo. A estos aprendientes probablemente les resultaría de mayor utilidad lanzarse a escribir, dejar que fluyan las ideas y luego involucrarse en una revisión más pormenorizada de su discurso. De lo anterior se desprende que el grado de aprovechamiento que se obtiene de la posibilidad de planificar la producción escrita podría estar sujeto al perfil del alumnado, al bagaje de conocimientos que posee y a las características particulares de las tareas implementadas.

Un segundo punto que precisa atención se relaciona con el factor subyacente a la variabilidad observada en la actuación, como consecuencia de las diferentes condiciones de implementación de las tareas utilizadas en la investigación empírica. Teniendo en cuenta la operacionalización de la planificación previa (10 minutos de planificación y tiempo delimitado para realizar la tarea) y la planificación en tiempo real (sin restricciones de tiempo), cabría preguntarse si es la planificación per se la que, según se ha sugerido en la bibliografía especializada, conduce a mejoras en cuanto a ciertos aspectos lingüísticos o si,

2. Ortega (2004) aborda de manera detallada las diferencias y similitudes entre las propuestas de estos autores. 
por otro lado, el tiempo total dedicado a la escritura constituye el factor más importante para la producción de un texto de calidad. Como sostienen Hayes y Gradwohl Nash (1996, en Al-Humaidi, 2008, p. 78), el tiempo que el escritor dedica a su texto parece ser un determinante más fuerte de la calidad de su discurso que la planificación per se.

Sumado a lo anterior, se ha de hacer hincapié en la necesidad de considerar la recursividad de los procesos de planificación, textualización y revisión en los estudios que examinan la interacción entre diferentes tipos de planificación y producción escrita, ya que la planificación puede tener lugar incluso en aquellas condiciones en las que el investigador se ha propuesto impedirlo. Asimismo, las mejoras que supuestamente conlleva la planificación previa podrían confundirse con aquellas mejoras que se deben a otros procesos que tienen lugar durante la escritura y dentro de los límites de tiempo establecidos para la realización de tareas concretas (revisar y hacer un rastreo visual para detectar errores, volver al primer fragmento, borrarlo y empezar desde el principio, etc.).

Partiendo de las reflexiones anteriores y considerando las dificultades que supone manipular la planificación en la expresión escrita — proceso este que puede darse en cualquier momento, en diferentes niveles y en relación con diferentes aspectos lingüísticos (Manchón, 2014)—, el presente estudio persigue examinar el impacto de la manipulación de la variable tiempo en la tarea [time-on-task] en la complejidad, corrección y fluidez de la expresión escrita de un grupo de aprendientes griegos de español como LE (ELE). Adicionalmente, se establecen comparaciones directas entre el discurso escrito de estos informantes y el de nativos de español en cuanto a las dimensiones lingüísticas de complejidad sintáctica y fluidez. Mediante esta comparación no se persigue evaluar la actuación de los primeros en términos de éxito o fracaso (Cook, 1999), sino más bien explorar en qué medida dicha actuación se ve afectada por los requerimientos adicionales que supone el procesamiento de una lengua diferente a la materna (Foster y Tavakoli, 2009; Kormos, 2011).

\section{Metodología}

\subsection{Participantes}

En el estudio participaron 65 aprendientes griegos de ELE del Instituto Cervantes de Atenas y del Centro de Idiomas Extranjeros de la Universidad Kapodistríaka (Atenas), de edades oscilantes entre los 20 y los 38 años $(M=26.26, S D=4.94$, Mediana=24), que se encontraban en un nivel intermedio (B2) ${ }^{3}$. La muestra de hablantes nativos estuvo constituida por 46 estudiantes del Máster Universitario en Lingüística Aplicada a la

\footnotetext{
3. En ambos centros, los aprendientes realizan un examen final de curso interno antes de acabar la última sesión de clase y, dependiendo de su nota, pasan al nivel siguiente. A pesar de que dicho examen carece de las propiedades de un examen de certificación y puede ser más flexible, los grupos formados por nivel se pueden considerar en gran medida homogéneos.
} 
enseñanza de ELE de la Universidad Nebrija (Madrid), de edades comprendidas entre los 23 y los 51 años $(M=32.2, S D=7$, Mediana $=31)$, nacidos en su mayoría en España (87\%) y con residencia también en España (67.4\%). La mayoría de ellos (70.5\%) eran profesores de lenguas (ELE, otra LE, castellano) y un 32.5\% tenía experiencia de más de 3 años en la enseñanza de ELE.

\subsection{Instrumentos}

Para los propósitos del presente estudio se utilizaron dos historietas del caricaturista argentino Quino (Apéndice 1), ambas utilizadas en estudios previos (la primera tarea en Mavrou, 2013, y la segunda tarea en Gilabert, 2005, 2007). La manipulación de su complejidad cognitiva se hizo en términos de la cantidad de tiempo que tendrían los informantes para su realización:

- Tarea 1 (T1): 10 minutos en total; el aprendiente se involucra en la producción escrita bajo presión de tiempo.

- Tarea 2 (T2): 20 minutos en total; el aprendiente realiza la tarea bajo condiciones relativamente más favorables de actuación.

Las decisiones tomadas al respecto se basaron en los siguientes procedimientos: (a) análisis cualitativo de las tareas (validez de contenido), (b) juicios de expertos, y (c) pilotaje de las tareas en el que participaron hablantes nativos de griego $(n=11$, solo T2) y español ( $n=5$, T1 y T2) y aprendientes griegos de ELE $(n=10)$. Los resultados de dichos procedimientos mostraron que las dos tareas podrían considerarse equivalentes en función de (1) una serie de criterios relacionados con las tareas en sí, las condiciones de su implementación y los recursos y procesos ejecutivos necesarios para su realización, y (2) una serie de medidas lingüísticas que se consideraron en el pilotaje (número de palabras, unidades terminales y cláusulas, longitud de la cláusula e índice de subordinación). Además, no hubo grandes discrepancias respecto al tiempo necesario para su realización (pilotaje) ni en la percepción subjetiva de su dificultad (pilotaje), la cual fue evaluada mediante dos escalas Likert de 9 opciones al igual que en otros estudios previos (Gilabert, 2005; Gilabert, Barón y Llanes, 2009; Stacey Shiau y Adams, 2011)4.

Por último, debe señalarse que el interés se centró en el discurso escrito desarrollado únicamente con tiempos verbales de pasado y con apoyo contextual a fin de lograr un mayor control sobre las condiciones experimentales del estudio.

\footnotetext{
4. No obstante, es necesario señalar que, al medir la percepción de la dificultad de las tareas una vez manipulado el tiempo para su realización $(n=65)$ y después de aplicar la prueba de rangos con signo de Wilcoxon, se hallaron diferencias estadísticamente significativas, siendo la T1 la que se percibió como más difícil $(Z=-2.38, p=.017)$. Este resultado sugiere que el modo de operacionalizar las tareas en términos de su complejidad cognitiva había sido adecuado.
} 


\subsection{Procedimiento de recogida de datos}

La recogida de las muestras escritas de los aprendientes de ELE se hizo en sus correspondientes centros de estudio de ELE, mientras que las muestras escritas de los hablantes nativos se recopilaron en la Universidad Nebrija. Todos los informantes que participaron en el estudio llevaron a cabo ambas tareas, las cuales se administraron utilizando la técnica de contrabalanceo. Las condiciones de implementación de estas y las instrucciones proporcionadas para su realización se mantuvieron idénticas para ambos grupos.

Una vez obtenido el corpus de producciones escritas, se procedió a su análisis de acuerdo con las 14 medidas lingüísticas presentadas en la Tabla 1.

TABla I: MEdidAS DE COMPLEJIDAD, CORRECCiÓN Y FLUIDEZ

\begin{tabular}{|c|c|c|}
\hline Variables & Medidas lingüísticas & Especificaciones \\
\hline \multirow[t]{3}{*}{$\begin{array}{l}\text { Complejidad } \\
\text { sintáctica }\end{array}$} & $\begin{array}{l}\text { 1. Índice de } \\
\text { Subordinación } \\
\text { (IS)=CL/UT }\end{array}$ & $\begin{array}{l}\text { Recomendada por Wolfe-Quintero, } \\
\text { Inagaki y Kim (1998) y Larsen-Freeman } \\
\text { (2006) para medir la complejidad } \\
\text { sintáctica del discurso escrito. }\end{array}$ \\
\hline & $\begin{array}{l}\text { 2. Complejidad Sintáctica } \\
\text { (CS1)=CLdep/UT }\end{array}$ & Medida específica de la complejidad sintáctica. \\
\hline & $\begin{array}{l}\text { 3. Complejidad Sintáctica } \\
\text { (CS2)=CLdep/CL }\end{array}$ & Medida específica de la complejidad sintáctica. \\
\hline \multirow[t]{3}{*}{$\begin{array}{l}\text { Complejidad } \\
\text { léxica }\end{array}$} & $\begin{array}{l}\text { 4. Índice de Uber } \\
\text { (IU)=Log²Types/ } \\
\text { Log(PAL/Types) }\end{array}$ & $\begin{array}{l}\text { Medida recomendada para evaluar la } \\
\text { diversidad léxica de textos breves (Jarvis 2001, } \\
\text { en Dewaele y Pavlenko, 2003, pp. 129-130). }\end{array}$ \\
\hline & $\begin{array}{l}\text { 5. Variación Léxica } \\
(\mathrm{VL})=\text { Typeslex/PALlex }\end{array}$ & $\begin{array}{l}\text { Medida aplicable a clases de palabras, } \\
\text { en este caso, los ítems léxicos. }\end{array}$ \\
\hline & $\begin{array}{l}\text { 6. Densidad Léxica } \\
\text { (DL)=PALlex/PAL }\end{array}$ & \\
\hline $\begin{array}{l}\text { Corrección } \\
\text { lingüística }\end{array}$ & 7. UTLE.UT=UTLE/UT & $\begin{array}{l}\text { Medida propuesta por Wolfe-Quintero et } \\
\text { al. (1998) y Larsen-Freeman (2006) para } \\
\text { evaluar la corrección lingüística del discurso } \\
\text { escrito. Para su cómputo se tomaron en } \\
\text { cuenta los errores morfosintácticos y léxicos. }\end{array}$ \\
\hline
\end{tabular}




\begin{tabular}{|c|c|c|}
\hline & 8. CLLE.CL=CLLE/CL & $\begin{array}{l}\text { Medida alternativa a la ratio anterior. Dado } \\
\text { que se basa en el concepto de CL, que } \\
\text { es una unidad de producción más breve, } \\
\text { resulta más fácil alcanzar un estatus libre de } \\
\text { errores (Ishikawa, 1995; Wolfe-Quintero et } \\
\text { al., 1998). Para su cómputo se tomaron en } \\
\text { cuenta los errores morfosintácticos y léxicos. }\end{array}$ \\
\hline & $\begin{array}{l}\text { 9. Porcentaje de } \\
\text { corrección lingüística } \\
(\mathrm{PPL})=[100-(\text { Err } \\
\text { x } 100 / \mathrm{PAL})]\end{array}$ & $\begin{array}{l}\text { Medida que expresa el porcentaje de } \\
\text { corrección lingüística en relación con } \\
\text { el número total de palabras. Para su } \\
\text { cómputo se tomaron en cuenta, además } \\
\text { de los errores morfosintácticos y léxicos, } \\
\text { todos los errores de acentuación. }\end{array}$ \\
\hline Fluidez & $\begin{array}{l}\text { 10. Número total de PAL } \\
\text { 11. Número total de UT } \\
\text { 12. Número total de CL }\end{array}$ & $\begin{array}{l}\text { Medidas que se basan en la frecuencia } \\
\text { absoluta de ciertas unidades de análisis } \\
\text { del discurso; su uso se considera adecuado } \\
\text { cuando se impone un límite de tiempo } \\
\text { para el desempeño de las tareas o se trata } \\
\text { de tareas del mismo tipo y de características } \\
\text { similares (Wolfe-Quintero et al., 1998). }\end{array}$ \\
\hline \multirow[t]{2}{*}{$\begin{array}{l}\text { Medidas de } \\
\text { longitud }\end{array}$} & $\begin{array}{l}\text { 13. Longitud de la UT } \\
(\mathrm{LUT})=\mathrm{PAL} / \mathrm{UT}\end{array}$ & \\
\hline & $\begin{array}{l}\text { 14. Longitud de la } \\
\text { CL }(\mathrm{LCL})=\mathrm{PAL} / \mathrm{CL}\end{array}$ & \\
\hline
\end{tabular}

Nota: UT=Unidad(es) Terminal(es) (toda oración o CL principal más todas las subordinadas o proposiciones insertadas en ella); $\mathrm{CL}=\mathrm{Cláusula(s)}$ (toda construcción sintácticamente libre o dependiente con el verbo en forma personal); CLdep=Cláusulas dependientes; PAL=Palabras; lex=Ítems de contenido referencial y semántico; UTLE=UT libres de errores; CLLE=CL libres de errores; Err=Errores.

\section{Resultados}

A fin de examinar el impacto de la manipulación de la variable tiempo en la tarea en la complejidad, corrección y fluidez lingüísticas, y después de comprobar la normalidad de los valores obtenidos (Tablas 2 y 3), se optó por la prueba T de Student para muestras relacionadas 5 . Dado que se efectuaron $t$-tests separados para cada una de las medidas

5. Los valores de asimetría y de curtosis para la mayoría de las medidas utilizadas estuvieron dentro del intervalo de $|-1+1|$, lo cual es indicativo del cumplimiento del supuesto de normalidad, y solo en muy pocos casos dichos valores superaron el 2 (solo curtosis). Según Wiedermann y von Eye (2013), varios estudios han mostrado que la prueba $\mathrm{T}$ de Student para muestras relacionadas es altamente sólida a violaciones del 
lingüísticas utilizadas, y con el objetivo de controlar la tasa de falsos positivos, se estableció un nivel crítico de $\alpha=.0036$ (ajuste de Bonferroni).

Tabla 2: Medidas lingüísticas utilizadas en la Ti (Aprendientes de ELE)

\begin{tabular}{|l|l|l|l|l|}
\hline & \multicolumn{1}{|c|}{ M } & \multicolumn{1}{c|}{ SD } & \multicolumn{1}{c|}{ Asimetría } & \multicolumn{1}{c|}{ Curtosis } \\
\hline T1IS & 1.39 & 0.22 & 1.29 & 2.14 \\
\hline T1CS1 & 0.40 & 0.22 & 1.18 & 2.07 \\
\hline T1CS2 & 0.27 & 0.10 & 0.15 & 0.23 \\
\hline T1IU & 16.21 & 2.52 & 1.22 & 2.23 \\
\hline T1VL & 0.77 & 0.08 & -0.18 & -0.32 \\
\hline T1DL & 0.42 & 0.03 & 0.42 & 0.98 \\
\hline T1UTLE.UT & 0.33 & 0.17 & 0.48 & 0.08 \\
\hline T1CLLE.CL & 0.41 & 0.18 & 0.17 & -0.60 \\
\hline T1PPL & 85.93 & 5.75 & -0.23 & -0.93 \\
\hline T1PAL & 111.71 & 27.65 & 1.11 & 1.26 \\
\hline T1UT & 12.71 & 3.62 & 0.67 & 0.44 \\
\hline T1CL & 17.37 & 4.67 & 0.63 & 0.08 \\
\hline T1LUT & 9.11 & 2.05 & 0.94 & 1.29 \\
\hline T1LCL & 6.56 & 1.09 & 0.82 & 0.99 \\
\hline
\end{tabular}

Tabla 3: Medidas lingüísticas utilizadas en la T2 (Aprendientes de ELE)

\begin{tabular}{|l|c|c|c|c|}
\hline & $\mathbf{M}$ & SD & Asimetría & Curtosis \\
\hline T2IS & 1.61 & 0.27 & 0.52 & 0.27 \\
\hline T2CS1 & 0.61 & 0.25 & 0.46 & 0.30 \\
\hline T2CS2 & 0.36 & 0.10 & -0.45 & 0.23 \\
\hline T2IU & 14.41 & 1.44 & 0.25 & -0.45 \\
\hline T2VL & 0.71 & 0.06 & -0.04 & 0.06 \\
\hline T2DL & 0.40 & 0.03 & 0.73 & 1.79 \\
\hline
\end{tabular}

supuesto de normalidad con respecto al error Tipo I, por lo que no se consideró necesario realizar los análisis sobre el número de observaciones resultante después de eliminar los casos atípicos. 


\begin{tabular}{|l|c|c|c|c|}
\hline T2UTLE.UT & 0.23 & 0.16 & 0.48 & -0.36 \\
\hline T2CLLE.CL & 0.38 & 0.17 & -0.08 & -0.40 \\
\hline T2PPL & 86.14 & 5.15 & -0.69 & 0.78 \\
\hline T2PAL & 139.23 & 36.93 & 1.30 & 2.37 \\
\hline T2UT & 12.82 & 3.71 & 0.67 & 0.76 \\
\hline T2CL & 20.34 & 5.86 & 0.60 & 0.69 \\
\hline T2LUT & 11.23 & 2.57 & 1.03 & 0.99 \\
\hline T2LCL & 7.01 & 1.23 & 0.57 & 0.29 \\
\hline
\end{tabular}

Los resultados de la comparación de medias (Tabla 4) mostraron que la manipulación de la complejidad cognitiva de las tareas tuvo un efecto moderado en el número total de CL $(t(64)=-4.6, p<.001, d=0.55)$ y grande en el número total de PAL $(t(64)=-7.94, p$ $<.001, d=0.81$ ), en la densidad de subordinación (IS: $t(64)=-5.74$; CS1: $t(64)=-6.02$; CS2: $t(64)=-6.01 ; p s<.001, d=0.89$ para IS, CS1 y CS2) y en la LUT $(t(64)=-6.89, p<.001$, $d=0.90$ ), siendo la T2 la que resultó con un mayor grado de elaboración sintáctica y de fluidez. Asimismo, se observaron diferencias estadísticamente significativas y considerables en las medidas de complejidad léxica, aunque estas fueron en la dirección contraria, es decir, el grado de diversidad léxica fue más elevado en la T1 (VL: $t(64)=5.57, p<.001$, $d=0.73$; IU: $t(64)=6.38, p<.001, d=0.83$, DL: $t(64)=3.38, p=.001, d=0.44)$. En lo referente a la corrección lingüística, se constató un impacto moderado de la manipulación de la complejidad de las tareas en la medida UTLE.UT $(d=0.56)$, siendo la actuación de los informantes más correcta en la T1, al menos con respecto a esta medida. Sin embargo, los mismos resultados no se replicaron para las medidas CLLE.CL y PPL.

TABla 4: Comparación de MEdias (ENTRE TAREAS)

DE LAS MEDIDAS DE COMPLEJIDAD, CORRECCIÓN Y FLUIDEZ

\begin{tabular}{|l|l|c|c|c|c|c|}
\hline & & M & SD & SE & $\boldsymbol{t}$ & $\boldsymbol{d}$ de Cohen \\
\hline Par 1 & T1IS \& T2IS & -2.19 & 0.31 & 0.38 & $-5.74^{* * *}$ & 0.89 \\
\hline Par 2 & T1CS1 \& T2CS1 & -0.21 & 0.28 & 0.03 & $-6.02^{* * *}$ & 0.89 \\
\hline Par 3 & T1CS2 \& T2CS2 & -0.09 & 0.12 & 0.02 & $-6.01^{* * *}$ & 0.89 \\
\hline Par 4 & T1VL \& T2VL & 0.05 & 0.08 & 0.01 & $5.57^{* * *}$ & 0.73 \\
\hline Par 5 & T1IU \& T2IU & 1.80 & 2.37 & 0.28 & $6.38^{*+*}$ & 0.83 \\
\hline Par 6 & T1DL \& T2DL & 0.02 & 0.04 & 0.004 & $3.38^{* * *}$ & 0.44 \\
\hline
\end{tabular}




\begin{tabular}{|l|l|c|c|c|c|c|}
\hline Par 7 & $\begin{array}{l}\text { T1UTLE.UT \& } \\
\text { T2UTLE.UT }\end{array}$ & 0.09 & 0.18 & 0.02 & $4.13^{* * *}$ & 0.56 \\
\hline Par 8 & $\begin{array}{l}\text { T1CLLE.CL \& } \\
\text { T2CLLE.CL }\end{array}$ & 0.02 & 0.18 & 0.02 & 1.002 & 0.13 \\
\hline Par 9 & T1PPL \& T2PPL & -1.13 & 5.55 & 0.69 & -1.64 & 0.19 \\
\hline Par 10 & T1PAL \& T2PAL & -27.52 & 27.95 & 3.47 & $-7.94^{* * *}$ & 0.81 \\
\hline Par 11 & T1UT \& T2UT & -0.11 & 3.54 & 0.44 & -0.25 & 0.003 \\
\hline Par 12 & T1CL \& T2CL & -2.97 & 5.21 & 0.65 & $-4.60^{* * *}$ & 0.55 \\
\hline Par 13 & T1LUT \& T2LUT & -2.12 & 2.50 & 0.31 & $-6.89^{* * *}$ & 0.90 \\
\hline Par 14 & T1LCL \& T2LCL & -0.45 & 1.21 & 0.15 & $-2.99^{* *}$ & 0.39 \\
\hline
\end{tabular}

Nota: ${ }^{* *} p \leq .01,{ }^{* * *} p \leq .001$

Con el objetivo de examinar si existen diferencias estadísticamente significativas entre el discurso escrito nativo y no nativo en cuanto a la complejidad sintáctica y la fluidez, y después de comprobar que se cumplían los supuestos de normalidad (Tabla 5) y de homocedasticidad (Tabla 6), se utilizó la prueba T de Student para muestras independientes ${ }^{6}$. La comparación de medias (Tabla 7) se hizo estableciendo un nivel crítico de $\alpha=.0031$ (ajuste de Bonferroni).

Según los resultados obtenidos, los hablantes nativos escribieron textos más elaborados sintácticamente (T1IS: $t(79.04)=-3.94, p<.001, d=0.89$; T2IS: $t(109)=-3.66, p<.001$, $d=0.70$; T1CS1: $t(77.19)=-3.91, p<.001, d=0.89$; T2CS1: $t(109)=-3.45, p=.001, d=0.67$; T1CS2: $t(109)=-3.87, p<.001, d=0.74$; T2CS2: $t(109)=-3.03, p=.003, d=0.58)$ y utilizaron UT y CL más largas en ambas tareas, y estas diferencias fueron grandes (T1LUT: $d=1.08$; T2LUT: $d=1.42$; T1LCL: $d=0.63$; T2LCL: $d=1.05$ ).

Tabla 5: Medidas lingüÚśsticas utilizadas en las Ti y T2 (Hablantes nativos)

\begin{tabular}{|l|c|c|c|c|}
\hline & M & SD & Asimetría & Curtosis \\
\hline T1PAL & 148.93 & 34.50 & 0.16 & -0.76 \\
\hline T2PAL & 172.37 & 36.18 & 0.32 & -0.36 \\
\hline
\end{tabular}

6. La evidencia empírica sugiere que dicha prueba es, por lo general, sólida ante moderadas violaciones del supuesto de normalidad, tamaños muestrales casi iguales y relativamente grandes y, en caso de heterogeneidad, menor varianza en el grupo de menor tamaño (Posten, 1984; Rasch y Guiard, 2004; Sawilowsky y Blair, 1992; Stonehouse y Forrester, 1998). 


\begin{tabular}{|l|c|c|c|c|}
\hline T1UT & 13.80 & 4.84 & 0.39 & -0.50 \\
\hline T2UT & 11.98 & 3.76 & 0.61 & 0.69 \\
\hline T1CL & 21.09 & 5.84 & 0.48 & -0.25 \\
\hline T2CL & 21.00 & 5.38 & 0.29 & 0.51 \\
\hline T1IS & 1.59 & 0.30 & 0.71 & 0.36 \\
\hline T2IS & 1.81 & 0.30 & 0.45 & 0.03 \\
\hline T1CS1 & 0.60 & 0.30 & 0.47 & -0.25 \\
\hline T2CS1 & 0.78 & 0.30 & 0.31 & -0.58 \\
\hline T1CS2 & 0.36 & 0.12 & -0.44 & 0.06 \\
\hline T2CS2 & 0.42 & 0.10 & -0.17 & 0.41 \\
\hline T1LUT & 11.68 & 3.65 & 1.17 & 1.76 \\
\hline T2LUT & 15.34 & 4.23 & 0.71 & 0.58 \\
\hline T1LCL & 7.27 & 1.45 & 0.68 & 0.10 \\
\hline T2LCL & 8.44 & 1.52 & 0.90 & 0.85 \\
\hline
\end{tabular}

Tabla 6: Prueba de LeVene y ratio de varianzas para las medidas de complejidad SINTÁCTICA y FLUIDEZ (APRENDIENTES de ELE y Hablantes NATIVOS)

\begin{tabular}{|l|c|c|c|c|}
\hline & $\begin{array}{c}\text { Estadístico } \\
\text { de Levene } \boldsymbol{F}\end{array}$ & $\begin{array}{c}\text { Varianza } \\
\text { Aprendientes ELE }\end{array}$ & $\begin{array}{c}\text { Varianza Hablantes } \\
\text { nativos }\end{array}$ & ${\text { Ratio } \text { Var }_{\text {max }} \text { /Var }_{\text {min }}}$ \\
\hline T1PAL & $4.37^{*}$ & 764.34 & 1190.15 & 1.56 \\
\hline T2PAL & 0.21 & 1363.99 & 1308.82 & 1.04 \\
\hline T1UT & $5.91^{*}$ & 13.09 & 23.41 & 1.79 \\
\hline T2UT & 0.00 & 13.75 & 14.11 & 1.03 \\
\hline T1CL & 1.49 & 21.83 & 34.08 & 1.56 \\
\hline T2CL & 0.31 & 34.35 & 28.89 & 1.19 \\
\hline T1IS & $5.28^{*}$ & 0.05 & 0.09 & 1.8 \\
\hline T2IS & 1.19 & 0.07 & 0.09 & 1.60 \\
\hline T1CS1 & $7.88^{* *}$ & 0.05 & 0.08 & 1.50 \\
\hline T2CS1 & 2.65 & 0.06 & 0.09 & 2 \\
\hline T1CS2 & 2.47 & 0.01 & 0.02 & 1 \\
\hline T2CS2 & 0.15 & 0.01 & 0.01 & \\
\hline
\end{tabular}




\begin{tabular}{|c|c|c|c|c|}
\hline T1LUT & $13.55^{* * *}$ & 4.18 & 13.34 & 3.19 \\
T2LUT & $12.77^{* * *}$ & 6.63 & 17.86 & 2.69 \\
\hline T1LCL & $4.90^{*}$ & 1.18 & 2.09 & 1.77 \\
\hline T2LCL & 0.95 & 1.51 & 2.31 & 1.53 \\
\hline
\end{tabular}

Nota: ${ }^{*} p<.05,{ }^{* *} p \leq .01,{ }^{* * *} p \leq .001$; Ratio $\operatorname{Var}_{\max } / \operatorname{Var}_{\min }<4$

Tabla 7: Comparación de MEdias (ENTRE GRupos)

DE LAS MEDIDAS DE COMPLEJIDAD SINTÁCTICA Y FLUIDEZ

\begin{tabular}{|l|c|c|c|c|c|c|c|}
\hline & \multicolumn{2}{|c|}{$\begin{array}{c}\text { Aprendientes } \\
\text { ELE }\end{array}$} & \multicolumn{2}{c|}{$\begin{array}{c}\text { Hablantes } \\
\text { Nativos }\end{array}$} & \multicolumn{2}{|l|}{} \\
\cline { 2 - 8 } & M & SD & M & SD & $t$ & $\boldsymbol{d}$ de Cohen & $\begin{array}{c}\text { r effect size } \\
\text { correlation }\end{array}$ \\
\hline T1PAL & 111.71 & 27.65 & 148.93 & 34.50 & $-6.07^{* * *}$ & 1.33 & .55 \\
\hline T2PAL & 139.23 & 36.93 & 172.37 & 36.18 & $-4.70^{* * *}$ & 0.91 & .41 \\
\hline T1UT & 12.71 & 3.62 & 13.80 & 4.84 & -1.30 & 0.29 & .14 \\
\hline T2UT & 12.82 & 3.71 & 11.98 & 3.76 & 1.17 & 0.22 & .11 \\
\hline T1CL & 17.37 & 4.67 & 21.09 & 5.84 & $-3.72^{* * *}$ & 0.71 & .34 \\
\hline T2CL & 20.34 & 5.86 & 21.00 & 5.38 & -0.61 & 0.12 & .06 \\
\hline T1IS & 1.39 & 0.22 & 1.59 & 0.30 & $-3.94^{* * *}$ & 0.89 & .41 \\
\hline T2IS & 1.61 & 0.27 & 1.81 & 0.30 & $-3.66^{* * *}$ & 0.70 & .33 \\
\hline T1CS1 & 0.40 & 0.22 & 0.60 & 0.30 & $-3.91^{* * *}$ & 0.89 & .41 \\
\hline T2CS1 & 0.61 & 0.25 & 0.78 & 0.30 & $-3.45^{* * *}$ & 0.67 & .31 \\
\hline T1CS2 & 0.27 & 0.10 & 0.36 & 0.12 & $-3.87^{* * *}$ & 0.74 & .35 \\
\hline T2CS2 & 0.36 & 0.10 & 0.42 & 0.10 & $-3.03^{* *}$ & 0.58 & .28 \\
\hline T1LUT & 9.11 & 2.05 & 11.68 & 3.65 & $-4.33^{* * *}$ & 1.08 & .47 \\
\hline T2LUT & 11.23 & 2.57 & 15.34 & 4.23 & $-5.88^{* * *}$ & 1.42 & .58 \\
\hline T1LCL & 6.56 & 1.09 & 7.27 & 1.45 & $-2.81^{* *}$ & 0.63 & .30 \\
\hline T2LCL & 7.01 & 1.23 & 8.44 & 1.52 & $-5.44^{* * *}$ & 1.05 & .46 \\
\hline & & & & & & & \\
\hline
\end{tabular}


En lo que concierne a las medidas de fluidez, si bien el grupo de hablantes nativos utilizó un mayor número de palabras en ambas tareas (T1PAL: $t(83.13)=-6.07, p<.001$, $d=1.33$; T2PAL: $t(109)=-4.7, p<.001 d=0.91)$ y de CL en la T1 $(t(109)=-3.72, p<.001$, $d=0.71$ ), diferencias estas que además de significativas resultaron grandes, las diferencias entre los dos grupos (nativos y no nativos) con respecto al número total de UT en las T1 y T2 y a la cantidad de CL empleadas en la T2 fueron mínimas según se comprobó mediante los tamańos del efecto.

\section{Discusión}

El primer objetivo del presente estudio fue examinar el impacto de la manipulación del tiempo dedicado a la realización de dos tareas escritas en las dimensiones lingüísticas de complejidad, corrección y fluidez.

Según los resultados obtenidos, la tarea de menor complejidad cognitiva (T2) potenció la producción de un discurso más fluido y elaborado sintácticamente en comparación con la T1 que se llevó a cabo bajo presión de tiempo, resultado que parcialmente concuerda con los obtenidos en otros estudios que se ocuparon de la producción oral (por ejemplo, Gilabert, 2005, 2007). Contar con tiempo suficiente para los procesos de escritura (como en el caso de la T2) supone las siguientes ventajas: mayor atención prestada a los procesos de codificación lingüística, mayor flexibilidad para atender a diferentes aspectos de la lengua y para experimentar y probar hipótesis lingüísticas (complejidad sintáctica), y facilitación de los procesos de búsqueda y recuperación, desde la memoria a largo plazo, de conocimiento declarativo relacionado con los mecanismos de vinculación sintáctica que rigen la lengua meta. En lo que respecta a la T1, el tiempo limitado para su realización probablemente restó a los aprendientes la posibilidad de proporcionar más detalles sobre los sucesos de la historieta (fluidez), de considerar y reflexionar sobre diferentes formas de expresar sus ideas o de atreverse a asumir riesgos (complejidad sintáctica), de ahí que sus textos presentaran un menor grado de elaboración sintáctica con oraciones más breves.

Otra explicación plausible se deriva de los postulados de la Hipótesis de Cognición (Robinson, 2001, 2003). Según esta hipótesis, un aumento de la complejidad cognitiva a lo largo de la dimensión resource-directing (narración en pasado) acompañado de una menor complejidad cognitiva en la dimensión resource-depleting (planificación del discurso o la posibilidad de contar con más tiempo para los procesos de escritura como en el caso de la T2) resultaría en una mayor complejidad sintáctica y mayor corrección. Los resultados del presente trabajo proporcionan evidencia parcial a esta predicción.

Ahora bien, es necesario hacer una puntualización. Tanto los hablantes nativos como los no nativos produjeron textos más fluidos y más elaborados sintácticamente a partir de la T2. En cuanto a la fluidez, se podría asumir que el mayor tiempo concedido para la 
realización de una tarea poco demandante a nivel de conceptualización (T2) se convirtió en una condición suficiente para garantizar la producción de textos más extensos. Esta afirmación está en consonancia con lo señalado por Hayes (2009), que los procesos de traducción de ideas y de textualización requieren una importante cantidad de tiempo. Sin embargo, en lo que se refiere a la complejidad sintáctica, es necesario considerar la posibilidad de que las tareas utilizadas no potenciaran un grado de complejidad sintáctica uniforme. De hecho, algunas vińetas de la T2 probablemente obligaron a la formulación de oraciones subordinadas al presentar un personaje en primer plano y otro en el fondo de la historieta, mientras que las vińetas de la T1 presentaban o un solo personaje o dos involucrados en la misma acción (Tavakoli y Foster, 2011), cuestiones estas que no se pudieron detectar en el pilotaje de las tareas, probablemente debido al número reducido de informantes que participaron en este.

Diferencias estadísticamente significativas se observaron también en la complejidad y densidad léxicas, aunque en la dirección contraria, es decir, los textos resultantes a partir de la T1 presentaron un vocabulario más variado y denso. Dos explicaciones podrían dar cuenta de estos resultados. En primer lugar, los textos generados a partir de la T2 presentaron un mayor grado de complejidad sintáctica, lo cual se traduce en el uso de un mayor número de palabras de clase cerrada para establecer la vinculación sintáctica (conjunciones, preposiciones, etc.). Manteniendo constante el número de palabras, un mayor uso de palabras funcionales resultaría en un menor número de palabras de contenido referencial y semántico.

En segundo lugar, es posible que la T1 fuese ligeramente más fácil en términos de carga lingüística, ya que los segmentos necesarios para traducir verbalmente los sucesos presentados en esta historieta (comer, beber, cena, cocina, etc.) se suelen enseñar y se adquieren en etapas más tempranas del aprendizaje de ELE en comparación con aquellos segmentos que requería la T2 (pasillo, cajera, carrito, etc.). El ensayo y uso repentino de los primeros probablemente potenció su recuperación más rápida y eficiente, independientemente de las condiciones de implementación de las dos tareas.

En lo que concierne a la corrección lingüística, el hecho de que no se observaran diferencias estadísticamente significativas entre las dos tareas podría atribuirse a la naturaleza asincrónica y a la recursividad de los procesos de escritura, que favorecen el automonitoreo, la autocorrección y la reelaboración del discurso (Al-Humaidi, 2008, y Farahani y Meraji, 2011, obtuvieron resultados similares; para resultados diferentes se remite a Meraji, 2011).

Alternativamente, si se asume que el grado de corrección alcanzado refleja el conocimiento lingüístico actual que posee el aprendiente de LE (Wolfe-Quintero et al., 1998), se podría suponer que la manipulación de la complejidad cognitiva de las tareas no es una condición suficiente por sí sola para promover y potenciar esta dimensión lingüística (Stacey Shiau y Adams, 2011), al menos en lo referente al discurso escrito. 
El segundo objetivo que se planteó fue comparar la actuación de los aprendientes de ELE con la de un grupo de hablantes nativos de español con respecto a la complejidad sintáctica y la fluidez. En cuanto a la primera variable, los resultados mostraron diferencias estadísticamente significativas y grandes entre los dos grupos en todas las medidas de complejidad sintáctica utilizadas (medidas de subordinación y de longitud) y en ambas tareas.

Griffin y Spieler (2006, en Véliz, Riffo, Hernández, Sáez y Sáez 2013, pp. 244-245) sostienen que la exposición a estructuras sintácticas influye en cómo los hablantes las producen - fenómeno denominado priming sintáctico—y esto podría explicar la mayor complejidad del discurso de estudiantes universitarios, puesto que ellos tienen mayor contacto y probablemente han tenido que utilizar con mayor frecuencia estructuras sintácticamente complejas. Dado que muchos de los hablantes nativos del presente estudio eran profesores involucrados en la enseñanza de lenguas, sería de esperar que contasen con una amplia práctica y preparación con estas estructuras, de ahí que su discurso presentara un mayor grado de complejidad sintáctica en comparación con el de los hablantes no nativos.

Asimismo, para el hablante nativo, acceder y recuperar segmentos desde su memoria a largo plazo y crear con estos fragmentos enunciados más o menos elaborados, basándose en el conocimiento intuitivo del sistema gramatical de su lengua materna (LM) que le ofrece muchas alternativas respecto a la manera de vinculación sintáctica (Ferreira y Engelhardt, 2006), es un proceso más bien automatizado. Por otro lado, para el hablante no nativo la recuperación de conocimiento declarativo constituye un proceso en gran medida controlado y lento, y la cantidad de alternativas a las que puede recurrir es bastante limitada. Estas reflexiones justificarían las diferencias observadas en la complejidad sintáctica entre hablantes nativos y no nativos.

En lo que se refiere a la fluidez, los resultados mostraron diferencias estadísticamente significativas entre los dos grupos solo en una medida, el número total de palabras utilizadas en ambas tareas. Dewaele (2002) señala que las diferencias individuales en la fluidez se determinan por la cantidad de conocimiento implícito y el grado de aceleración en cuanto al uso del conocimiento explícito. El estatus incompleto de las representaciones lingüísticas del hablante no nativo y la mayor dependencia del conocimiento declarativo y explícito de la lengua meta (Dewaele, 2002) le obligan a involucrarse en un procesamiento controlado, que es lento y serial (Schneider y Chein, 2003). En cambio, el hablante nativo puede beneficiarse del conocimiento implícito de su LM para llevar a cabo tareas lingüísticas y, como señala Paradis (2000, en Dewaele, 2002, p. 231), acelerar el control sobre las reglas explícitas no es lo mismo que el uso automático de la competencia implícita.

Schneider y Chein (2003) afirman que el procesamiento automático no supone mucho esfuerzo y puede operar incluso en situaciones de alta carga cognitiva, mientras que el procesamiento controlado requiere un esfuerzo sustancial e interfiere con otras tareas que 
también demandan procesamiento controlado. Las dos tareas utilizadas en el presente estudio exigían el uso de un vocabulario específico, aunque este podría considerarse de alta frecuencia. Sería de suponer que, para un hablante nativo adulto, los procesos de acceso y recuperación de este vocabulario se llevan a cabo de manera automática, ya que estos segmentos forman parte del conocimiento implícito y procedimental de su LM. Por tanto, interferirían solo mínimamente con otros procesos y aspectos de la producción escrita y difícilmente podrían alterarse por distractores o por condiciones de actuación estresantes. Todo ello indudablemente garantiza una mayor fluidez discursiva en la LM.

Por otro lado, para el aprendiente de una LE, los procesos de acceso y recuperación de segmentos desde la memoria a largo plazo, la monitorización del output y la necesidad de alternar entre los dos modos de procesamiento, automático y controlado, consumen una gran cantidad de recursos atencionales y de memoria. La fluidez en este caso puede verse afectada por la dificultad que entrańa ejecutar los diferentes procesos de escritura de manera simultánea y por la necesidad de suprimir posibles interferencias generadas por la activación de segmentos conceptualmente equivalentes o fonológicamente similares a los de las demás lenguas que habla el aprendiente (como el inglés, idioma hablado por casi todos los informantes del presente estudio, y el francés estudiado por la mitad de ellos). Asimismo, el procesamiento lingüístico del hablante no nativo puede corromperse ante la presencia de distractores o bajo situaciones de alta carga cognitiva. Esto podría explicar los mayores tamaños del efecto que se observaron en cuanto al número de palabras producidas entre nativos y no nativos en la tarea de mayor complejidad cognitiva (T1).

\section{Conclusiones}

Mediante el presente estudio ha sido posible llegar a dos conclusiones: (a) proporcionar tiempo a los aprendientes de LE para que se involucren en los procesos de producción escrita y elegir cuidadosamente las características de las tareas implementadas son, al menos, dos de las condiciones necesarias para estimular la producción de un discurso elaborado sintácticamente; (b) dirigir la atención de los aprendientes hacia la corrección lingüística probablemente requiere instrucción explícita (Hulstijn y Hulstijn, 1984) y, tal vez, aún más tiempo.

Dichas conclusiones, sin embargo, deben considerarse con precaución debido a una serie de limitaciones inherentes al diseño metodológico del presente estudio. En concreto, lo ideal habría sido que los informantes realizaran las dos tareas también en su LM a fin de poder llegar a conclusiones más firmes con respecto al procesamiento lingüístico que subyace en la producción escrita en LM y LE. Del mismo modo, habría sido especialmente interesante optar por un diseño cruzado, con la participación de un grupo de informantes que realizara la T1 con más tiempo y la T2 con menos tiempo, y así poder controlar de modo más eficaz la variable de interés para el presente estudio. Otra limitación se refiere al tamaño muestral, el cual fue moderado, así como 
al hecho de que en el estudio participaran solo aprendientes de un nivel intermedio, lo cual limita el potencial de generalizar los resultados a la población meta. En cuanto al grupo de hablantes nativos, a pesar de que su número fue también moderado, la principal limitación tiene que ver con su composición. En otras palabras, habría sido conveniente contar con hablantes nativos de un rango de edades más variado y de un nivel de educación equiparable al de los hablantes no nativos. Poder superar todas estas limitaciones en futuras investigaciones permitiría dar mayor refuerzo a los resultados aquí obtenidos.

\section{Referencias}

Al-Humaidi, M. (2008). Planning in the writings of Saudi EFL learners at the College of Languages and Translation at KSU (Trabajo final de máster no publicado). King Saud University.

Cook, V. (1999). Going beyond the native speaker in language teaching. TESOL Quarterly, 33(2), 185-209. doi:10.2307/3587717

Dewaele, J. M. (2002). Individual differences in L2 fluency: The effect of neurobiological correlates. En V. Cook (Ed.), Portraits of the L2 user (pp. 219250). Clevedon: Multilingual Matters.

Dewaele, J. M., y Pavlenko, A. (2003). Productivity and lexical diversity in native and non-native speech: A study of cross-cultural effects. En V. Cook (Ed.), Effects of the second language on the first (pp. 120-141). Clevedon: Multilingual Matters.

Farahani, A. A. K., y Meraji, S. R. (2011). Cognitive task complexity and L2 narrative writing performance. Journal of Language Teaching and Research, 2(2), 445-456. doi: $10.4304 /$ jltr.2.2.445-456

Ferreira, F., y Engelhardt, P. E. (2006). Syntax and production. En M. J. Traxler y M. A. Gernsbacher (Eds.), Handbook of psycholinguistics (2a ed., pp. 61-92). Ámsterdam: Elsevier.

Foster, P., y Tavakoli, P. (2009). Native speakers and task performance: Comparing effects on complexity, fluency and lexical diversity. Language Learning, 59(4), 866-896. doi:10.1111/j.1467-9922.2009.00528.x

Gilabert, R. (2005). Task complexity and L2 narrative oral production (Trabajo final de máster no publicado). Universitat de Barcelona.

Gilabert, R. (2007). The simultaneous manipulation of task complexity along planning time and +/- Here-and-Now: Effects on L2 oral production. En M. P. García Mayo (Ed.), Investigating tasks in formal language learning (pp. 44-68). Clevedon: Multilingual Matters.

Gilabert, R., Barón, J., y Llanes, A. (2009). Manipulating cognitive complexity across task types and its impact on learners' interaction during oral performance. 
International Review of Applied Lingusitics in Language Teaching, 47, 367-395. doi: $10.1515 /$ iral.2009.016

Hayes, J. R. (2009). From idea to text. En R. Beard, D. Myhill, J. Riley y M. Nystrand (Eds.), The SAGE handbook of writing development (pp. 65-79). Londres: SAGE.

Hulstijn, J. H., y Hulstijn, W. (1984). Grammatical errors as a function of processing constraints and explicit knowledge. Language Learning, 34(1), 23-43. doi:10.1111/j.1467-1770.1984.tb00994.x

Ishikawa, S. (1995). Objective measurement of low-proficiency EFL narrative writing. Journal of Second Language Writing, 4(1), 51-69. doi:10.1016/10603743(95)90023-3

Kormos, J. (2011). Task complexity and linguistic and discourse features of narrative writing performance. Journal of Second Language Writing, 20(2), 148-161. doi: 10.1016/j.jslw.2011.02.001

Larsen-Freeman, D. (2006). The emergence of complexity, fluency, and accuracy in the oral and written production of five Chinese learners of English. Applied Linguistics, 27(4), 590-619. doi:10.1093/applin/aml029

Manchón, R. M. (2014). The internal dimension of tasks: The interaction between task factors and learner factors in bringing about learning through writing. En $\mathrm{H}$. Byrnes y R. M. Manchón (Eds.), Task-based language learning - Insights from and for L2 writing (pp. 27-52). Ámsterdam: John Benjamins.

Mavrou, I. (2013). Precisión lingüística y complejidad sintáctica: ¿̨hasta qué punto compiten entre sí por los recursos limitados del aprendiz? Revista Nebrija de Lingüistica Aplicada, 14, 75-90.

Meraji, S. R. (2011). Planning time, strategy use, and written task production in a pedagogic vs. a testing context. Journal of Language Teaching and Research, 2(2), 338-352. doi:10.4304/jltr.2.2.338-352

Ortega, L. (2004). Aproximaciones cognitivo-interaccionistas al aprendizaje de segundas lenguas mediante tareas. Estudios de Lingüistica Inglesa Aplicada, 5, 1540. Recuperable en: http://institucional.us.es/revistas/elia/5/1.ortega.pdf

Posten, H. O. (1984). Robustness of the two-sample t-test. En D. Rasch y M. L. Tiku (Eds.), Robustness of statistical methods and nomparametric statistics (pp. 9299). Dordrecht: Reidel.

Rasch, D., y Guiard, V. (2004). The robustness of parametric statistical methods. Psychology Science, 46(2), 175-208.

Robinson, P. (2001). Task complexity, cognitive resources, and syllabus design: A triadic framework for examining task influences on SLA. En P. Robinson (Ed.), Cognition and second language instruction (pp. 287-318). Cambridge: Cambridge University Press.

Robinson, P. (2003). The Cognition Hypothesis, task design, and adult task-based language learning. Second Language Studies, 21(2), 45-105. 
Robinson, P. (2007). Criteria for classifying and sequencing pedagogic tasks. En M. P. García Mayo (Ed.), Investigating tasks in formal language learning (pp. 7-27). Clevedon: Multilingual Matters.

Sawilowsky, S. S., y Blair, R. C. (1992). A more realistic look at the robustness and type II error properties of the $t$ test to departures from population normality. Psychological Bulletin, 111(2), 352-360. doi:10.1037/0033-2909.111.2.352

Schneider, W., y Chein, J. M. (2003). Controlled \& automatic processing: behavior, theory, and biological mechanisms. Cognitive Science, 27(3), 525-559. doi:10.1016/S0364-0213(03)00011-9

Skehan, P. (1996). A framework for the implementation of task-based instruction. Applied Linguistics, 17(1), 38-62. doi:10.1093/applin/17.1.38

Skehan, P. (1998). A cognitive approach to language learning. Oxford: Oxford University Press.

Stacey Shiau, Y. S., y Adams, R. (2011). The effects of increasing reasoning demands on accuracy and complexity in L2 oral production. University of Sydney Papers in TESOL, 6, 121-146.

Stonehouse, J. M., y Forrester, G. J. (1998). Robustness of the $\mathrm{t}$ and U tests under combined assumption violations. Journal of Applied Statistics, 25(1), 63-74. doi:10.1080/02664769823304

Tavakoli, P., y Foster, P. (2011). Task design and second language performance: The effect of narrative type on learner output. Language Learning, 61(S1), 37-72. doi:10.1111/j.1467-9922.2011.00642.x

Véliz, M., Riffo, B., Hernández, M., Sáez, Y., y Sáez, K. (2013). Oraciones producidas por adultos mayores y adultos jóvenes en una situación controlada. Onomázein, 27, 241-257.

Wiedermann, W. T., y von Eye, A. (2013). Robustness and power of the parametric $\mathrm{t}$ test and the nonparametric Wilcoxon test under non-independence of observations. Psychological Test and Assessment Modeling, 55(1), 39-61.

Wolfe-Quintero, K., Inagaki, S., y Kim, H. Y. (1998). Second language development in writing: Measures of fluency, accuracy \& complexity (Technical Report 17). Honolulu, HI: University of Hawai'i, Second Language Teaching and Curriculum Center. 


\section{Apéndice: Tareas escritas}
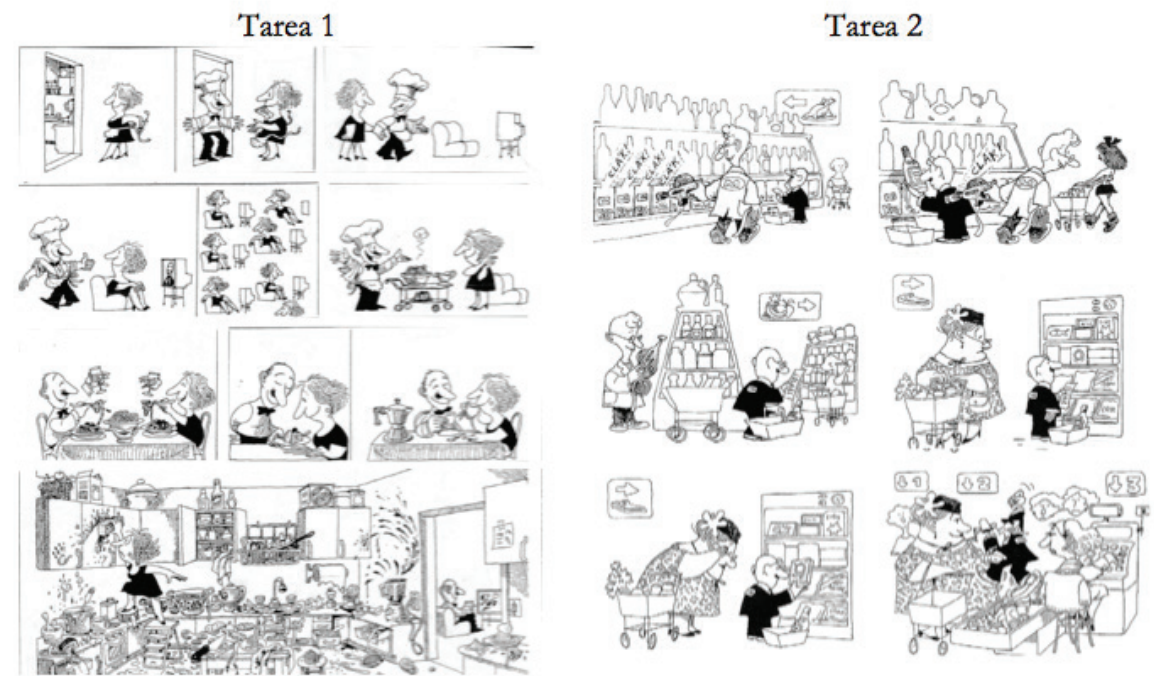